\title{
UTILISATION DE LA TECHNIQUE DE DIGESTIBILITÉ IN VITRO POUR PRÉVOIR LA VALEUR ALIMENTAIRE DES FOURRAGES
}

\author{
M. CHENOST (1) \\ avec Ia collaboration technique de Marinette JAILLER \\ Station de Recherches sur l'Élevage des Ruminants, \\ Centre de Recherches de Clermont-Ferrand, 63-Saint-Genès-Champanelle. \\ Institut national de la Recherche agronomique
}

\section{RÉSUMÉ}

La technique de fermentation in vitro mise au point par TILLEY et TERRY (I96r) permet de suivre la cinétique de la digestion des plantes fourragères. La digestibilité de la matière sèche obtenue après de courtes durées de fermentation ( 8 heures pour les fourrages verts, 24 heures pour les foins) est en relation avec les quantités ingérées. La précision de la prévision est du même ordre que celle obtenue par les auteurs ayant utilisé la digestibilité $i n$ vitro de la cellulose.

Le produit des digestibilités in vitro $8 \times 4^{8}$ heures et 24 heures $\times 4^{8}$ heures permet de prévoir la quantité de matière organique digestible ingérée qui caractérise le mieux la valeur alimentaire des fourrages. La précision de l'estimation est meilleure pour les foins que pour les fourrages verts et pour les premiers cycies que pour les repousses. Elle est un peu moins bonne que celle permise par la méthode des sachets de nylon mais elle est aussi bonne que celle obtenue à partir de la digestibilité in vivo et de toute façon meilleure que celle obtenue à partir de la teneur en cellulose brute.

\section{INTRODUCTION}

La valeur alimentaire d'un fourrage dépend de la quantité qui peut en être ingérée et de sa valeur énergétique, dont le meilleur critère est le coefficient de digestibilité de la matière organique.

De très nombreux chercheurs ont cherché à prévoir la digestibilité ; ils ont essentiellement utilisé des techniques chimiques et, depuis $5_{5}$ ans, des techniques de diges-

(1) Adresse actuelle : Station de Recherches zootechniques, Centre de Recherches agronomiques des Antilles et de la Guyane, Domaine Duclos, Petit-Bourg, Guadeloupe, Institut national de la Recherche agronomique. 
tion in vitro par le jus de rumen (PIGDEN et BeLL, I955 cf. revue de Johnson, I966). En revanche, très peu se sont intéressés à la prévision des quantités ingérées.

Celles-ci dépendent non seulement de la digestibilité (Br,AXTER et al., I96I ; DEMARquIrLY, I965) et des caractères d'appétibilité du fourrage (par exemple sa teneur en parties mortes, Demarquin, y, I965) mais essentiellement de la vitesse avec laquelle il est digéré dans le rumen (DONFFER et al., I960; MIrFord et Minson, I965; Demarquiliy et Chenost, I969). Pour prévoir la quantité de fourrage ingérée il faut donc mesurer celle-ci. C'est ainsi que DoneFFr et al. (I96o) avaient proposé d'utiliser la technique de fermentation in vitro pendant des durées courtes et qu'ils avaient relié la quantité ingérée à la digestibilité in vitro I2 heures de la cellulose.

Dans le même esprit nous avons pensé que la digestibilité in vitro de la matière sèche des fourrages, mesurée au bout d'une courte période ( 12 ou 24 heures) pourrait présenter une liaison intéressante avec la quantité ingérée, alors que celle mesurée au bout d'une plus longue durée ( 48 heures) nous permet de caractériser la digestibilité. Nous avons utilisé la technique de TILLEY et TERRY (I963) qui est une des plus satisfaisantes (cf. BARNES, I 967 ).

\section{MATÉRIEI ET MÉTHODES}

\section{Fourrages}

Nous avons utilisé 68 fourrages verts et 57 foins représentant différentes espèces qui ont été récoltées aux différents cycles de végétation. Les graminées récoltées au premier cycle de croissance constituent 55 p. roo de chacune de ces deux catégories de fourrages. La digestibilité et la quantité ingérée ont été mesurées sur moutons par DEMARQUILLY ; elles varient respectivement de $5 \mathrm{I}, 4$ à 82,2 p. Ioo et de 47,2 à $94,0 \mathrm{~g} / \mathrm{kg} \mathrm{P}^{0,75}$ pour les graminées (Ray-grass d'Italie, Fétuque élevée et Dactyle) et de 53,6 à 79,4 p. I oo et 42,2 à $100,5 \mathrm{~g} / \mathrm{kg} \mathrm{P} \mathrm{P}^{0,75}$ pour les légumineuses (Trèfle violet et Luzerne). La digestibilité des fourrages verts a été mesurée directement sur le fourrage frais coupé chaque matin.

Les échantillons sur lesquels nous avons travaillé ont été constitués à partir du fourrage offert pendant la période de mesure de la digestibilité. Ils ont été séchés à l'étuve à $80^{\circ} \mathrm{C}$ pendant 24 heures et broyés (grille à maille de $0,8 \mathrm{~mm}$ de diamètre). On a dosé leurs teneurs en cendres, en matières azotées et en cellulose brute Weende.

\section{Fermentation in vitro}

Nous avons utilisé la technique de fermentation in vitro de TILLEy et TERRY (I963); les durées de fermentation choisies ont été : 8,24 et 48 heures. L'animal donneur est une vache adulte tarie porteuse d'une large fistule du rumen et consommant une ration de bon foin de luzerne. L'échantillon du jus de rumen est prélevé, à l'aide d'une sonde de $50 \mathrm{~cm}$ munie d'une crépine, 3 heures après la distribution du premier repas de la journée.

Les résultats de fermentation que nous présentons ont été corrigés après ajustement des séries entre elles grâce à deux foins standards, l'un de graminée (d'une digestibilité de la matière sèche de $65, \circ$ p. roo), l'autre de luzerne (d'une digestibilité de la matière sèche de 56,8 p. roo) utilisés dans chaque série.

Pour simplifier l'exposé des résultats, nous utiliserons dans la suite du texte les abréviations suivantes :

D. I. V. : digestibilité in vitro de la matière sèche.

D. I. V. R. : digestibilité in vitro du résidu insoluble dans l'eau à $40^{\circ} \mathrm{C}$ (calculée à partir de la teneur en résidu insoluble dans l'eau).

M. I. S. : quantité de matière sèche ingérée.

M. O. D. I. : quantité de matière organique digestible ingérée. 


\section{RÉ,SUL,TATS}

Choix de la durée de la fermentation in vitro

Nous avons vérifié que la technique de fermentation in vitro permettait bien de mettre en évidence des différences dans la vitesse de dégradation des fourrages.

Pour cela nous avons fait fermenter pendant des périodes de 8,24 et 48 heures deux groupes de fourrages. Le premier est constitué de 2 graminées et de 2 luzernes de digestibilité comprise entre $5 \mathrm{I}$, o et $53,3 \mathrm{p}$. Ioo; le deuxième de 3 graminées et de 2 luzernes de digestibilité comprise entre 59,I et 63,3 p. Ioo. Dans chaque groupe les fourrages sont de digestibilité très voisine et ont été choisis parce qu'ils avaient été ingérés en quantité très différente : de 37,5 à $6 \mathrm{I}, \mathrm{I} \mathrm{g} / \mathrm{kg} \mathrm{P}^{\mathbf{0}, 75}$ pour le premier groupe et de 40,9 à $6 \mathrm{I}, 3 \mathrm{~g} / \mathrm{kg} \mathrm{P}^{0,25}$ pour le deuxième groupe.

Dans chaque groupe les D. I. V., après 48 heures de fermentation, sont très voisines ; celles obtenues après 8 heures et 24 heures sont différentes et se classent sensiblement dans le même ordre que les M. S. I. (fig. r).

Il existe également des différences entre fourrages dans les D. I. V. R. obtenus après 8 et 24 heures de fermentation. Ces derniers se classent également dans le même ordre que les quantités de matière sèche ingérées (fig. I).

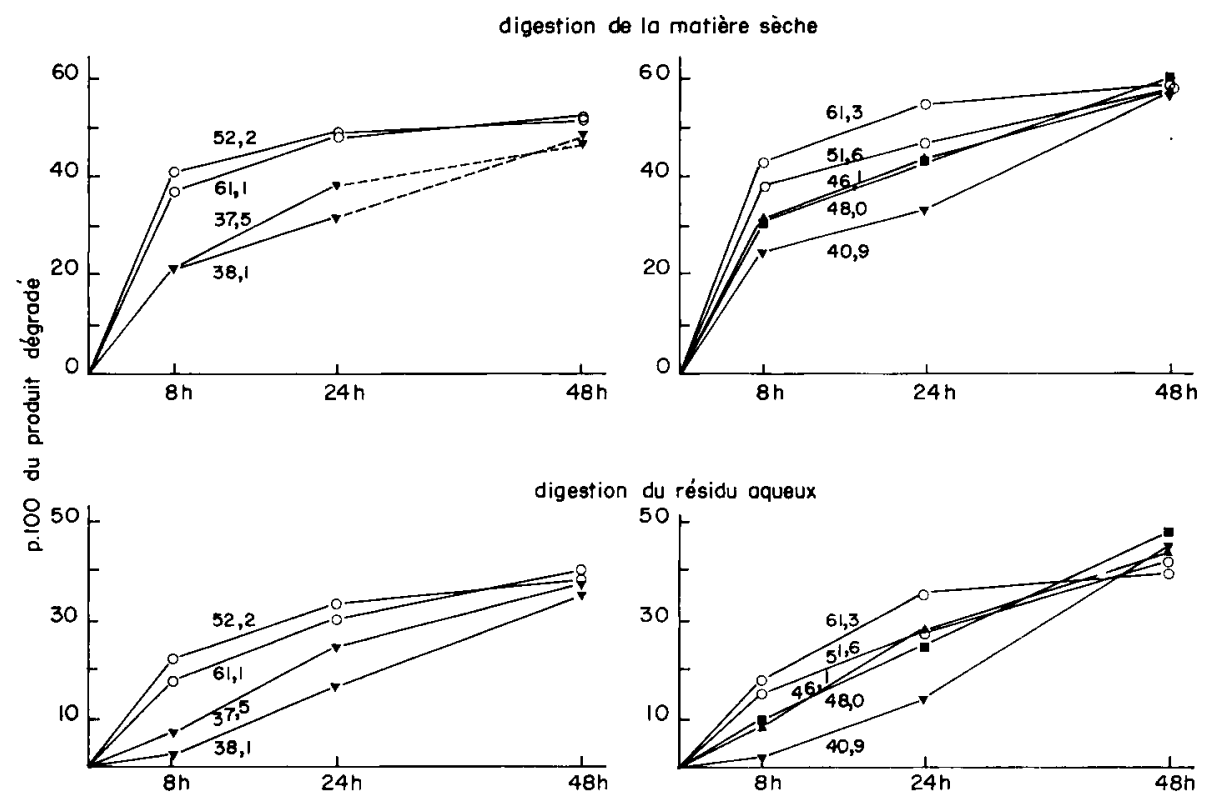

FIG. I. - Cinétique de la digestion in vitro de la matière sèche et du résidu aqueux de deux groupes de fourrages de digestibilité voisine, ingêrés en quantités différentes

(la quantité de matière sèche ingérée est portée sur chaque courbe correspondante)

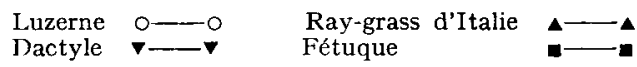


Nous avons alors cherché sur un nombre de fourrages plus élevé ( 26 foins et I 8 fourrages verts) lequel des deux critères, D. I. V. ou D. I. V. R., il convenait de prendre pour estimer la quantité ingérée et après quelle durée de fermentation ce critère donnait l'estimation la plus précise (tabl. I). C'est la D. I. V. qu'il convient de choisir et c'est après 8 heures de fermentation pour les fourrages verts et 24 heures pour les foins que la M. S. I. présente les liaisons les plus étroites avec la D. I. V.

Nous pouvons en outre estimer la digestibilité des fourrages en prolongeant la durée des fermentations jusqu'à 48 heures.

\section{TABI.EAU I}

Relation entre la quantité de matiève sèche ingérée et la digestibilité in vitro : infuence de la durée de fermentation et du substrat

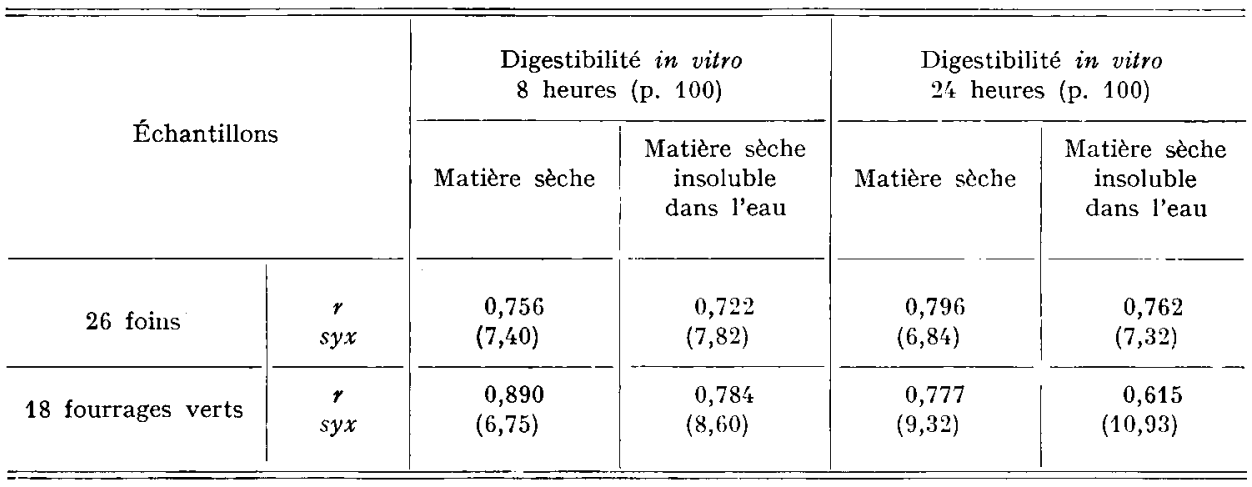

\section{Relation avec la valeur alimentaire}

Nous avons utilisé les critères de fermentation in vitro définis précédemment pour voir avec quelle précision ils permettent d'estimer la M. S. I., la digestibilité et la M. O. D. I de l'ensemble de notre échantillonnage comportant 68 fourrages verts et 57 foins.

La M. S. I. du foin est estimée par 1a D. I. V. 24 heures avec une meilleure précision (l'écart type réduit $s y x=6,89$ ) que celle des fourrages verts par la D.I.V. 8 heures $(s y x=8,75)$ (tabl. 2). La digestibilité in vitro est un meilleur critère d'estimation de la M. S. I. que la cellulose brute, ou, même, que la digestibilité in vivo (tabl. 3). Elle ne permet pas, toutefois, de prévoir la M. S. I. de façon aussi précise que la digestibilité en sachets (DEMARQUILLY et CHEnosT, I969) où syx $=6,22$ et syx $=7,29$ respectivement pour les foins et les fourrages verts.

Pour chaque catégorie de fourrages la digestibilité est estimée à partir de la D. I. V. avec une meilleure précision que la M. S. I. Cette estimation est plus précise pour les foins $(s y x=2,75)$ que pour les fourrages verts $(s y x=4,7 \mathrm{I})$, chez lesquels elle est d'ailleurs moins bonne qu'avec la cellulose brute. Dans le cas des fourrages verts, l'estimation est plus précise pour les légumineuses que pour les graminées (tabl. 2).

Les foins ont une D. I. V. plus élevée que les fourrages verts de même digestibilité $i n$ vivo sauf dans le cas des légumineuses (fig. 2). Nous avons porté sur la figure 2 les équations de régression liant la digestibilité et la M. S. I. à la D. I. V. 
DIGESTIBIIITÉ " IN VITRO 》 DES FOURRAGES

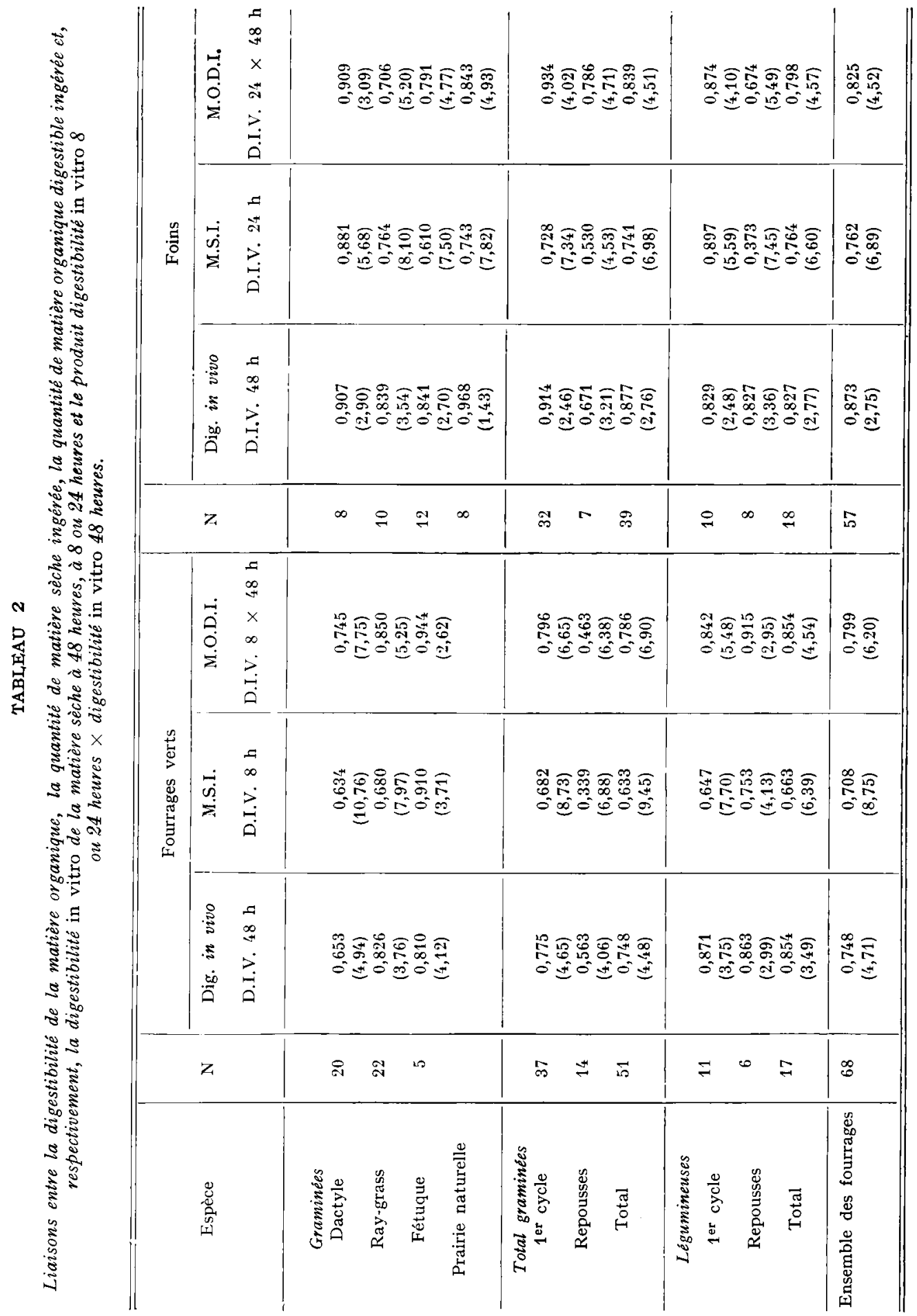


La quantité de M. O. D. I. des foins est estimée avec une bonne précision par le produit D. I. V. 24 heures $\times$ D. I. V. 48 heures notamment pour les foins de Dactyles $(s y x=3,09)$ et pour les premiers cycles : syx $=4,02$ pour les graminées, $s y x=4$, $\mathrm{xo}$ pour les légumineuses (tab1. 3). Le fait que la précision soit meilleure pour les premiers cycles que pour les repousses peut s'expliquer facilement; en effet au cours du premier cycle la M. O. D. I. dépend essentiellement du stade de croissance du fourrage.

\section{TABLEAU 3}

Comparaison, pour l'ensemble des fourrages de cycles et d'espèces différentes, de l'estimation de la digestibilité, de la quantité ingérée et de la valeur alimentaire à partir des coefficients de digestibilité in vitro et de la teneur en cellulose brute

\begin{tabular}{|c|c|c|c|c|}
\hline \multirow[t]{2}{*}{ Critère d'estimation } & \multicolumn{2}{|c|}{$\begin{array}{c}\text { Fourrages verts }(n=68) \\
r\end{array}$} & \multicolumn{2}{|c|}{$\underset{r}{\text { Foins }}\left(n=\frac{57)}{s y x}\right.$} \\
\hline & \multicolumn{4}{|c|}{ Digestibilité de la matière organique } \\
\hline \multirow[t]{2}{*}{$\begin{array}{l}\text { D.I.V. } 48 \mathrm{~h} \ldots \ldots \ldots \ldots \ldots \ldots \\
\text { Cellulose brute } \ldots \ldots \ldots \ldots \ldots \ldots\end{array}$} & $\begin{array}{l}+0,748 \\
-0,802\end{array}$ & $\begin{array}{l}4,71 \\
4,09\end{array}$ & $\begin{array}{r}+0,872 \\
-0,716\end{array}$ & $\begin{array}{l}2,75 \\
3,95\end{array}$ \\
\hline & \multicolumn{4}{|c|}{ Quantité de matière sèche ingérée } \\
\hline 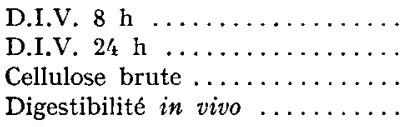 & $\begin{array}{l}+0,708 \\
-0,558 \\
+0,688\end{array}$ & $\begin{array}{r}8,75 \\
10,04 \\
9,11\end{array}$ & $\begin{array}{r}+\quad 0,762 \\
-\quad 0,618 \\
+\quad 0,580\end{array}$ & $\begin{array}{l}6,89 \\
9,21 \\
9,25\end{array}$ \\
\hline Digestibilité in vivo .......... & \multicolumn{4}{|c|}{ Quantité de matière organique digestible ingérée } \\
\hline D.I.V. 8 h $\ldots \ldots \ldots \ldots \ldots \ldots$ & $+0,731$ & 7,11 & & \\
\hline D.I.V. $2 \div h \ldots \ldots \ldots \ldots$ & & & $+0,72 \cdot \mathbf{3}$ & 5,77 \\
\hline D.I.V. $48 \mathrm{~h} \ldots \ldots \ldots \ldots \ldots$ & $+0,672$ & 7,77 & $+0,728$ & 5,47 \\
\hline D.I.V. $8 \times 48$ h. $\ldots \ldots \ldots \ldots \ldots$ & $+0,799$ & 6,90 & & \\
\hline D.I.V. $24 \times 48 \mathrm{~h} \ldots \ldots \ldots \ldots$ & & & $+0,825$ & 4,52 \\
\hline Cellulose brute $\ldots \ldots \ldots \ldots \ldots$ & $-0,713$ & 7,39 & $-0,724$ & 5,79 \\
\hline Digestibilité in vivo .......... & $+0,852$ & 5,42 & $+0,800$ & $4,7 / \mathbf{t}$ \\
\hline
\end{tabular}

Tout critère (constituant chimique, coefficient de digestibilité in vivo ou in vitro) lié à ce stade de croissance sera en même temps un bon critère de la M. O. D. I. En revanche, dans le cas des repousses, la valeur alimentaire dépend moins de l'âge et de la quantité ingérée mais va dépendre aussi des facteurs externes (teneur en matière sèche, souillures, etc.) ; il est donc beaucoup plus difficile de la prévoir.

La M. O. D. I. des fourrages verts est estimée par le produit D. I. V. $8 \mathrm{~h} \times$ D. I. V. $48 \mathrm{~h}$ avec une moins bonne précision que celle des foins par le produit D. I. V. $24 \times 48 \mathrm{~h}$, sauf dans le cas des légumineuses dont l'échantillonnage, il est vrai, est plus restreint $(\mathrm{N}=\mathrm{I} 7)$. Si la précision de l'estimation est meilleure pour les foins que pour les fourrages verts, c'est sans doute parce que les facteurs d'appétibilité (teneur en matière sèche, présence de parties mortes) sont plus importants pour les fourrages verts que pour les foins. 
D'une façon générale, l'estimation de la M. O. D. I. à partir du produit de deux D. I. V. est toujours plus précise que celle obtenue à partir de la cellulose brute ou de la D. I. V. 48 h (tabl. 3) ; elle est plus précise que celle obtenue à partir de la digestibilité in vivo seulement pour les foins (tab1. 3). La figure 3 et le tableau 2 montrent
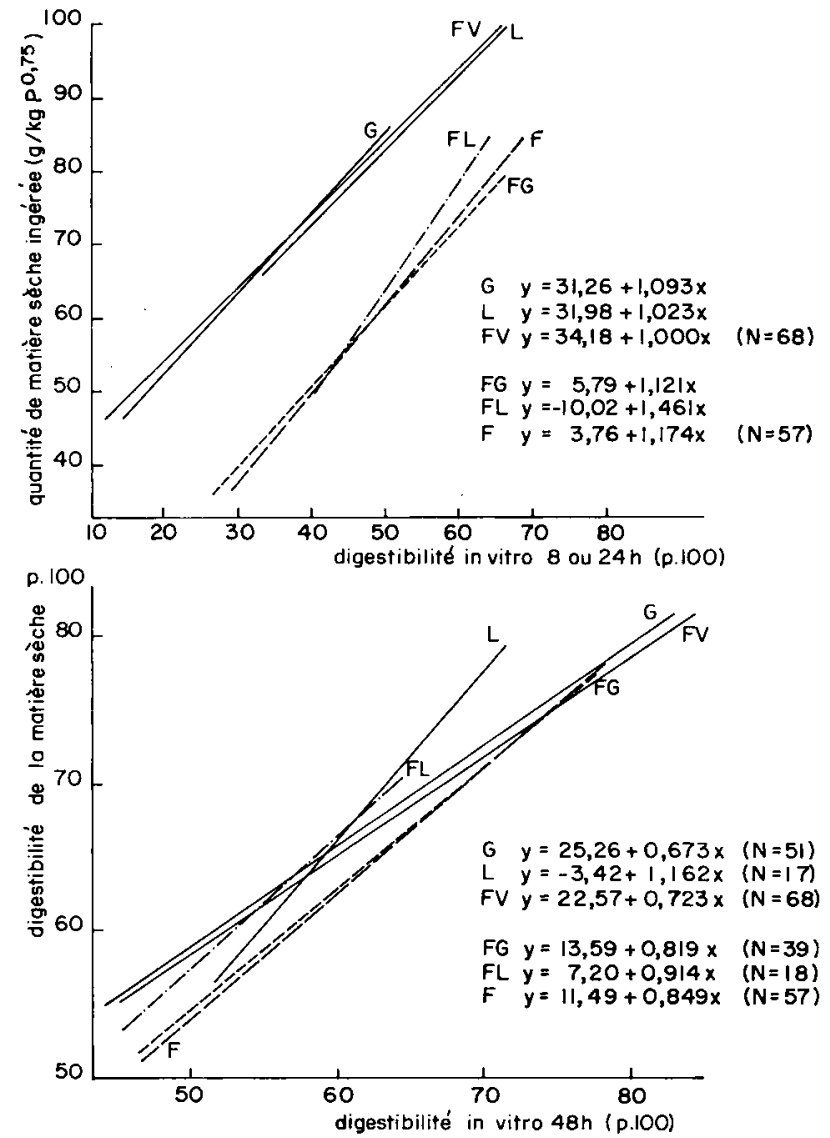

FIG. 2. - Relations entre la quantite de matière sèche ingerée et la D. I. V., 8 ou 24 heures et entre la digestibilité in vivo et la $D . I$. V. 48 heures

$$
\begin{aligned}
\mathrm{G} & =\text { fourrages verts de graminées } \\
\mathrm{L} & =\text { fourrages verts de légumineuses } \\
\mathrm{FV} & =\text { ensemble des fourrages verts } \\
\mathrm{FG} & =\text { foins de graminées } \\
\mathrm{FL} & =\text { foins de légumineuses } \\
\mathrm{F} & =\text { ensemble de foins }
\end{aligned}
$$

qu'à l'intérieur des catégories foins et fourrages verts, la liaison ne semble pas dépendre de la famille botanique; nous n'avons donc calculé qu'une équation de régression pour chacune de ces catégories entre le M. O. D. I. $(y)$ et le produit D. I. V. 8 ou $24 \mathrm{~h} \times$ D. I. V. $48 \mathrm{~h}(x)$.

$$
\begin{aligned}
& y=2,89+\mathrm{I}, 064 x \text { pour les foins }(r=0,825) . \\
& y=\mathrm{I} 5,90+\mathrm{I}, \mathrm{I} 70 x \text { pour les fourrages verts }(r=0,799) \text { (fig. 3). }
\end{aligned}
$$


La précision de l'estimation de la M. O. D. I. est un peu moins bonne qu'avec la technique des sachets de nylon (DEMARquiliy et Chenost, I969) $: \boldsymbol{r}=+0,793$ $(\mathbf{N}=54)$ pour les foins ; $r=+0,894(\mathrm{~N}=63)$ pour les fourrages verts.

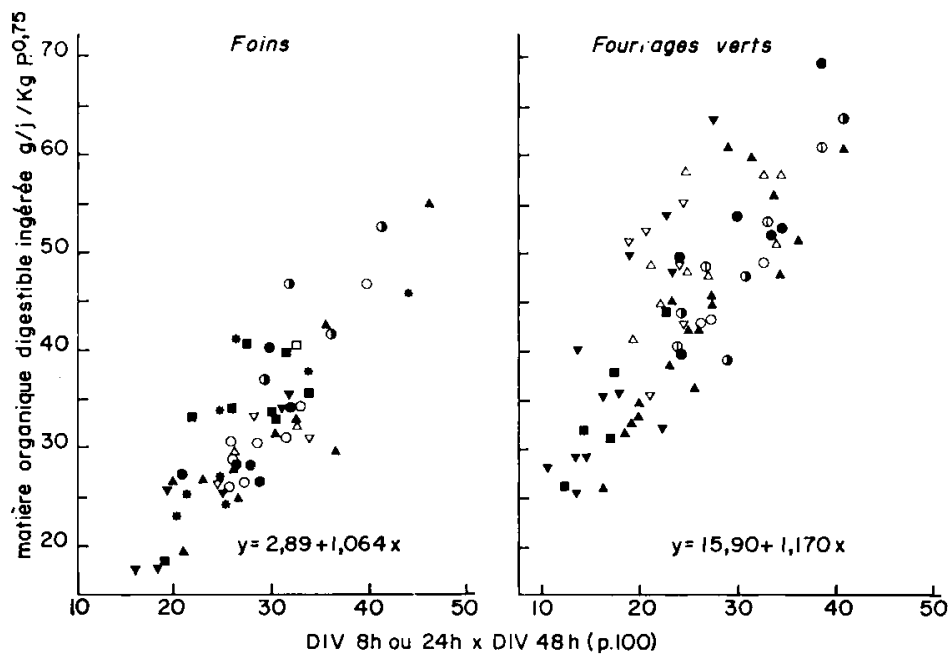

FIG. 3. - Liaison entre la quantité de matière organique digestible ingérée et le produit D. I. V. 8 heures ou 24 heures $\times D . I . V .48$ heures Ier cycle Repousses

\begin{tabular}{lll}
\multicolumn{1}{c}{-} & & - \\
Luzerne & $\circ$ & $\circ$ \\
Trèfle & $\mathbf{0}$ & $\mathbb{1}$ \\
Ray-grass & $\mathbf{\Delta}$ & $\Delta$ \\
Dactyle & $\mathbf{v}$ & $\nabla$ \\
Fétuque & $\mathbf{D}$ \\
Prairie naturelle & $*$ & $\square$
\end{tabular}

\section{Reproductibilité}

Pour mesurer la reproductibilité entre séries et intra séries de la digestibilité in vitro, nous avons soumis à une analyse de variance les résultats des digestions in vitro $8 \mathrm{~h}, 24 \mathrm{~h}, 48 \mathrm{~h}$ (conduites en triple) de 3 fourrages qui différaient par leur origine botanique et leur digestibilité, effectuées une fois par semaine pendant 3 semaines consécutives.

L'analyse de variance révèle des différences significatives entre séries, différences qui dépendent d'ailleurs de la durée de fermentation. Les coefficients de variation entre séries sont les plus faibles pour une durée de 24 h (4,7 p. roo) et les plus élevés pour une durée de $48 \mathrm{~h}$ (7,7 p. roo).

L'analyse de variance ne révèle pas de différences significatives intra séries entre les triples suivant la durée de fermentation ou la nature des fourrages. Les coefficients de variation intra série diminuent de $3, \mathrm{I}$ p. Ioo pour une durée de $8 \mathrm{~h}$ à I,9 p. Ioo pour une durée de 48 heures.

Ces résultats sont voisins de ceux des auteurs ayant suivi la cinétique de dégradation in vitro de la cellulose vraie (DONEFER, I960 ; CHALUPA, I966; KARN, JOHNSON, DEHORITY, I967) ou de la matière sèche (KARN et al., I967). La reproductibilité 
de la D. I. V. 48 h est cependant moins bonne que celle observée par TirLEY et TERRY (rg62) mais elle est voisine de celle observée par BARNEs (I967) qui comparait plusieurs techniques de fermentation in vitro.

\section{DISCUSSION}

Par le produit de deux D. I. V. mesurées pendant des durées différentes nous pouvons prévoir la quantité de matière organique digestible ingérée avec une précision comparable à celle obtenue par les chercheurs qui ont utilisé le produit de deux coefficients de digestibilité in vitro de la cellulose (dosée par la méthode de CRAMPTON et MAYNARD, I938) : DONEFER et al. ( $\mathrm{I} 960$ ), avec 3 foins de légumineuses et 2 foins de graminées, Arroyo (I963) avec 9 foins de graminées et Chalupa (I966) avec 25 foins de graminées.

Nos résultats présentent l'avantage d'avoir été obtenus d'une part sur un échantillonnage plus important et plus complexe, d'autre part à partir de la D. I. V. de la matière sèche. Cette mesure est plus simple et doit mieux caractériser la vitesse de digestion de 1'ensemble du fourrage.

Le fait de suivre la dégradation in vitro de la cellulose pour tenter de la relier à la quantité ingérée peut être discuté. En effet, la vitesse de digestion de la cellulose dépend de la rapidité d'attaque de l'ensemble de la membrane, cette rapidité dépendant elle-même de la structure et de la lignification de la membrane (Tomı,IN, I96I ; DEHORITY et al., I962). Cependant la vitesse de digestion d'un fourrage ne dépend pas seulement de la teneur en membranes et des caractéristiques de celles-ci; elle dépend également des proportions des différents constituants cytoplasmiques qui constituent le milieu de fermentation du rumen et qui sont entièrement digérés. On néglige celles-ci quand on relie la quantité ingérée à la seule dégradation in vitro de la cellulose.

C'est vraisemblablement pour ces raisons que, à la différence des autres méthodes, la relation entre la quantité ingérée et la D. I. V. 8 h est la même pour les graminées et les légumineuses dont les teneurs en constituants solubles sont pourtant très différentes, et que la précision d'estimation de la quantité ingérée est moins bonne avec 1a D. I. V. de la matière sèche insoluble dans l'eau qu'avec la D. I. V. de la matière sèche.

D'ailleurs KARN et al. (I967) observent effectivement une amélioration de la précision de l'estimation de la quantité ingérée avec la D. I. V. $8 \mathrm{~h}$ de la matière sèche par rapport à celle obtenue avec la D. I. V. 8 h de la cellulose.

Ia digestibilité in vitro est plus satisfaisante que le taux de cellulose brute pour prévoir la valeur alimentaire des fourrages mais cet avantage est pour l'instant compromis par une reproductibilité insuffisante.

Nous devons encore comparer cette méthode à d'autres techniques qui ont été mises au point à la Station : indice de fibrosité (CHENOST, résultats non publiés), résidu de la dégradation du fourrage par une cellulase (JARRIGE et THIVEND, I969) qui, elles, ne nécessitent pas la présence d'animaux porteurs de fistules du rumen. 


\title{
SUMMARY
}

\author{
PREDICTION OF FORAGE FEEDING VALUE BY MEANS \\ OF THE IN VITRO DIGESTIBILITY TECHNIQUE
}

Io We used the in vitro fermentation technique of TILLEY and TERRY (1962) to evaluate the forage dry matter intake by sheep and consequently the digestible organic matter intake.

$2^{\circ}$ A previous assay on 2 groups of forages of similar digestibility, but of different ingestion levels (fig. I) showed that after 8 or 24 hours of formentation, the in vitro digestibilities were generally classified in the same way as the dry matter intake.

$3^{\circ}$ The most suitable fermentation time to predict the dry matter intake is 8 hours for the green forages and 24 hours for the different types of hay (table I).

$4^{\circ}$ For 68 green forages and 57 types of hay (table 2), we calculated the relationships between I $^{\circ}$ the digestibility of the organic matter and the I. V. D. D. M. after 48 hours (fig. 2), $2^{\circ}$ the dry matter intake and the I. V. D. D. M. after 8 or 24 hours (fig. 2), and $3^{\circ}$ the digestible organic matter intake and the product of I. V. D. D. M. 48 hours $\times$ I. V. D. D. M. 8 or 24 hours (fig. 3). These data were compared with those obtained from the crude fibre content (Weende) (table 3).

$5^{\circ}$ The dry matter and digestible organic intake matter may generally be evaluated from the in vitro digestibility, the accuracy of estimation being higher for hay than for green forage (table 2) and higher for green forage from the first growing period than for the regrowth. This is probably due to a smaller influence of the external factors of the palatability (soil contamination, dead materials...).

$6^{\circ}$ The digestible organic matter intake may be evaluated from the product of the two in vitro digestibilities. The accuracy, which is satisfactory ( $s_{y x}$ generally inferior to 5,0 ), is better than that obtained from the in vitro digestibility after $4^{8}$ hours or from the crude fibre content (table 3).

\section{RÉFÉRENCES BIBLIOGRAPHIQUES}

Arroyo, Aguilu J. A., Evans J. L., Taylor M. W., I963. The artificial rumen technique for estimating the nutritive value of forages. J. of Agro. of the University of Puerto Rico, 47, I69-179.

Barnes R. F., I967. Collaborative in vitro rumen fermentation studies on forage substrates. J. Anim. Sci., 26, II20-I I 30 .

Blaxter I. L., Wainman F. W., Wilson R. S., rg6r. The regulation of food intake by sheep. Anim. Prod., 3, 5 I.

Chapula W., LeE D. D., 1966. Estimation of forage nutritive value from in vitro cellulose digestion. J. Dairy Sci., 49, I88-I92.

Crampton E. W., Maynard L. A., I938. The relation of cellulose and lignin content to the nutritive value of animal feeds. J. Nutr., 15, 383 .

Dehority B. A., Johnson R. R., Conrad H. R., ig62. The digestibility of forage hemicellulose and pectin by rumen bacteria in vitro and the effect of lignification thereon. J. Dairy Sci., 45, 508-5I2.

Demarquilly C., r965. Factors affecting the voluntary intake of green forage by sheep. Proc. 9th Intern. Grassland Congress. São Paulo. Section 9, o. p. 400 pp. 877-885.

Demarouilly C., Chenost M., r969. Étude de la digestion des fourrages dans le rumen par la méthode des sachets de nylon. Liaison avec la valeur alimentaire des fourrages. Ann. Zootech., 18, 4I9-436.

Donefer E., Crampton E. W., Lloyd L. E., r96o. Prediction of the nutritive value index of a forage from in vitro rumen fermentation data. J. Anim. Sci., 19, 545-552.

KARN J. F., Johnson R. R., Dehority B. A., I967. Rates of in vitro cellulose and dry matter digestion at 5,8 and II hours as predictors of forage nutritive value. J. Anim. Sci., 26, 381-384.

Milford, Minson D. J., I965. Intake of tropical pasture species. Proc. 9th Intern. Grassland Congress. São-Paulo pp. 8I5-822. 
Pigden W. J., Bell J. M., I955. The artificial rumen as a procedure evaluating forage quality. $J$. Anim. Sci., 14, I239 (Abstr.).

Tilley J. A. M., TERRY R. A., 1963. A two technique for in vitro digestion of forage crops. $J . B r$. Grassl. Soc., 18, I04-III.

Tomlin D. C., Dehority B. A., Johnson R. R., I96r. Differential effect of lignification in grasses and legumes on in vitro cellulose digestibility. J. Anim. Sci., 20, 963 (Abst.). 